Olmedo Moreno, Eva Ma (2013). Enfoques de aprendizaje de los estudiantes y metodología docente: Evolución hacia el nuevo sistema de formación e interacción propuesta en el EEES. Revista de Investigación Educativa, 31 (2), 411-429. http://dx.doi.org/10.6018/rie.31.2.133501

\title{
ENFOQUES DE APRENDIZAJE DE LOS ESTUDIANTES Y METODOLOGÍA DOCENTE: EVOLUCIÓN HACIA EL NUEVO SISTEMA DE FORMACIÓN E INTERACCIÓN PROPUESTA EN EL EEES
}

\author{
Eva $M^{\mathrm{a}}$ Olmedo Moreno \\ Universidad de Granada
}

\section{RESUMEN}

El gran desafío de la convergencia Europea en Educación Superior está en conseguir estructuras más flexibles y un nuevo enfoque basado en el aprendizaje. Este nuevo modelo de universidad se centra en una concepción del aprendizaje constructivista, en el que el estudiante es eje central en la creación de significado y el docente mediador entre el conocimiento y el alumnado.

Desde esta perspectiva, se pretende analizar la evolución y el proceso de aprendizaje en la Facultad de Ciencias de la Educación de la Universidad de Granada, en titulaciones conformadas desde la Ley de Reforma Universitaria (LRU), en experimentación con el Sistema de Transferencia de Créditos Europeo (ECTS) y en los actuales Grados. Determinándose qué aspectos cambiar o potenciar para alcanzar el nuevo modelo, no sólo a nivel teórico y general, sino también a nivel práctico y particular, se traza una línea de evolución en los modelos metodológicos docentes y de aprendizaje.

Palabras clave: Enfoques de aprendizaje, modelos metodológicos docentes, educación superior, cultura de aprendizaje.

\footnotetext{
Correspondencia:

Eva Mª Olmedo Moreno. Profesora Titular de Universidad. Dpto. Didáctica y Organización Escolar, Facultad de Ciencias de la Educación. Universidad de Granada. Campus Cartuja s/n 18071 - Granada. E-mail: emolmedo@ugr.es
} 


\title{
LEARNING APPROACHES OF STUDENTS AND TEACHING METHODOLOGY: DEVELOPMENT TOWARDS A NEW INTERACTION AND TRAINING SYSTEM INTHE ESHE
}

\begin{abstract}
The great challenge for European convergence in higher education lies in setting up more flexible structures and a new approach to learning. The concept of a new university is based on a constructivist conception of learning, in which the student plays a key role in creating meaning, and the teacher is a mediator between students and knowledge.

This study analyzed the evolution and the learning processes of undergraduate students in the Faculty of Education at the University of Granada, Spain. More specifically, we examined courses introduced under the LRU (University Reform Law) system and under the ECTS system, as well as current degree courses. Also, we identified which aspects ought to be changed or enhanced in order to accomplish the objectives of the new model, not only at a theoretical and general level, but also in practice; and developed teaching and learning methodology models.
\end{abstract}

Keywords: Learning approaches, teaching methodology, higher education, learning culture.

\section{INTRODUCCIÓN}

Actualmente, en la universidad nos encontramos con una propuesta de cambio metodológico y conceptual del aprendizaje en nuestras titulaciones. Estamos ante una concepción del aprendizaje constructivista, basada en el aprendizaje significativo, donde el estudiante es autónomo, auto-regulado, que conoce sus propios procesos cognitivos y tiene en sus manos el control del aprendizaje. La enseñanza, tanto en la teoría como en la práctica, ya no es una cuestión de transmitir conocimientos, recepcionarlos exactamente, almacenarlos y emplearlos de forma apropiada, sino más bien, el significado creado por el propio sujeto que aprende, no impuesto por la realidad o transmitido por enseñanza directa (Biggs, 1996).

Desde el constructivismo (Cole, 1990), el estudiante es eje central en la creación de significado y el docente mediador entre el conocimiento y el estudiante, generador de significado. Aunque puede parecer innovadora esta teoría del aprendizaje, tiene una larga tradición en la psicología cognitiva, sobre todo en niveles de primaria y secundaria. Sin embargo, en niveles universitarios, aunque no es novedosa en teoría, si que lo es en la práctica. El modelo EAC (Entornos de Aprendizaje Constructivista), desarrollado por Jonassen y Rorher-Murphy (1999) en la Universidad de Pensilvannia, es un buen ejemplo, puesto que trata de que el conocimiento sea elaborado individualmente y socialmente por los estudiantes, a través de sus propias experiencias y representaciones del mundo y sobre la base de los conocimientos declarativos ya conocidos.

Tanto en este modelo, como en otros, desarrollados desde enfoque constructivista, se fomenta la creación de contextos de aprendizaje basados en la comunicación, potenciando la creación de grupos de elaboración del conocimiento que tienen como objeto ayudar a los alumnos a "buscar el aprendizaje como finalidad de forma activa 
y estratégica" (Scardamalia y otros, 1994, p. 201), espacios de intercomunicación (en red, principalmente) y comunidades de aprendizaje de alumnado.

Existen trabajos previos que indagan en las concepciones de estrategias de aprendizaje en alumnos/as pertenecientes a dos contextos culturales distintos (Occidental y Oriental), como los de Dahlin y Watkins (2000), donde estudiantes, de ambas culturas, al enfrentarse ante un mismo proceso de aprendizaje lo hacen de forma particular y diferenciada. Tal es el caso del empleo de la memorización; que es un medio para llegar a la comprensión desde el punto de vista del alumnado occidental, o un mecanismo de repetición y esfuerzo para descubrir nuevos significados del material de estudio, desde una cultura oriental. Otros como Eklund-Myrskog (1998), han encontrado diferencias en orden al conocimiento básico, y a preferencias de evaluación (Birenbaum, 1997), de estudiantes universitarios de distintas titulaciones, debido principalmente al programa académico en el que se encuentran.

Tomando como referencia los trabajos anteriormente citados junto con las modalidades de aprendizaje establecidas por la CIDUA (2005), de la CRMEU (2006), De Miguel (2005 y 2006) y Cano y otros (2011), se realiza este estudio, que trata de conocer los Enfoques de aprendizaje del estudiante universitario ante determinados modelos formativos, para determinar qué elementos se encuentran vinculados a este nuevo modelo, y cómo establecer un programa de implementación de cambio el nuevo EEES eficaz.

Los Enfoques de aprendizaje vienen determinados por la predisposición del alumnado, en cuanto a su adaptación al contexto de aprendizaje, las competencias a alcanzar, y el modelo formativo, entre otros. Para Biggs (1987), está basado en un motivo o intención, que marca la dirección general de aprendizaje, y una estrategia o conjunto de estrategias, que seguiría esa dirección general. Siguiendo a Hernández (2000 y 2005), dadas unas metas que el estudiante ha de alcanzar, la autopercepción de su habilidad, la metodología de enseñanza, la evaluación y los resultados obtenidos, le servirán para que, tras un período de exposición a un marco de aprendizaje concreto, desarrolle un determinado Enfoque que le permita llevar a cabo sus tareas académicas lo más cómodamente posible.

Los modelos metodológicos docentes, llevan implícitos un tipo y una forma de docencia al igual que ésta implica un modo de evaluación. Existen múltiples clasificaciones de los modelos metodológicos en Educación Superior, aunque todos coinciden en establecer al menos dos tipos: tradicional o cuantitativo, basado en un aprendizaje cuantitativo, y el alternativo o cualitativo/constructivista, donde el aprendizaje se basa en la comprensión, significado y la construcción de un marco conceptual por el propio estudiante. Biggs (1996), establece un tercer tipo al que denomina estratégico, en el que el aprendizaje necesita ser validado por la institución. En este trabajo se ha tomado dicha clasificación como referencia, junto con la realizada por Porlán (1998), en la que se subdivide el modelo estratégico en: modelo tecnológico, donde el aprendizaje es mecánico e instrumental, y modelo espontaneísta, donde el aprendizaje está vinculado a la experimentación para llegar al conocimiento, aprender procedimientos y actitudes. 


\section{PROBLEMAY OBJETIVOS}

Desde este marco de referencia y en base a estudios previos realizados (Buendía y Olmedo, 2000; Buendía, Olmedo y Pegalajar, 2001; Buendía y Olmedo, 2003; Buendía, Olmedo y González, 2009), en esta investigación nos marcamos los siguientes objetivos para describir la posible relación entre los Enfoques de aprendizaje y los modelos metodológicos docentes, y su evolución en la universidad actual:

1. Identificar el Enfoque de aprendizaje de los estudiantes de la Facultad de Ciencias de la Educación, en tres momentos: titulaciones de LRU, en experimentación ECTS y nuevos de Grados.

2. Describir las principales características de los diferentes programas formativos que han cursado el alumnado de los grupos seleccionados, e identificarlos con un modelo metodológico docente.

3. Comparar los Enfoques de aprendizaje en los grupos seleccionados, en función del modelo metodológico docente, estableciendo sus respectivas líneas evolutivas.

\section{PARTICIPANTES}

La muestra de 648 estudiantes ha sido seleccionada, mediante un proceso polietápico, en función de la titulación y plan de estudios en el que se encuentran (Tabla 1). De manera que se establecen tres grupos: sistema de formación LRU, en experimentación con créditos ECTS, y en nuevos Grados. De los dos primeros grupos se seleccionan cuatro titulaciones, y se encuesta a un solo grupo de ellas elegido al azar, durante el mes de mayo de los cursos académicos 2005/06 para las titulaciones del programa formativo LRU y 2006/2007 para las de experimentación ECTS. Y en el tercer grupo, esto es, los nuevos Grados implementados durante el curso 2010/2011, se encuestan a las cuatros titulaciones existentes, seleccionando de forma proporcional los grupos en función del número en cada Grado.

Para el estudio de los modelos metodológicos docentes, se seleccionan 135 guías de las materias troncales y obligatorias/básicas (Tabla 2), de las titulaciones seleccionadas. 
TABLA 1

SELECCIÓN MUESTRAL

\begin{tabular}{|c|c|c|c|c|c|c|c|}
\hline & TITULAC & & 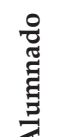 & 足 & 胥 & 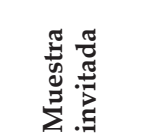 & 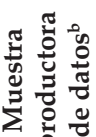 \\
\hline & Audic & y Lenguaje & 90 & 1 & 90 & -- & -- \\
\hline & Edu & ión Especial & 90 & 1 & 90 & -- & -- \\
\hline & & ación Física & 80 & 2 & 160 & -- & -- \\
\hline 胥 & $\begin{array}{r}\text { Educ } \\
\text { (Experime }\end{array}$ & $\begin{array}{l}\text { ón Infantil } \\
\text { ción ECTS) }\end{array}$ & 90 & 2 & Grupo A y B & Grupo A & 88 \\
\hline$\stackrel{U}{\ll}$ & Edu & ión Musical & 90 & 1 & 180 & -- & -- \\
\hline & Educación I & naria (LRU) & 100 & 3 & Grupo A, B y C & Grupo B & 90 \\
\hline & Lengua & Inglés & 100 & 1 & 100 & -- & -- \\
\hline & Extranjera & Francés & 50 & 1 & 50 & -- & -- \\
\hline & EDUCA & ŚN SOCIAL & 90 & 1 & 90 & -- & -- \\
\hline & $\begin{array}{r}\text { PEDAGOGÍA } \\
\text { LRU }\end{array}$ & $2^{\circ} \mathrm{CICLO}$ & 100 & 3 & Grupo A, B y C & Grupo A & 90 \\
\hline & $\begin{array}{r}\text { PSICO } \\
\text { (Experime }\end{array}$ & $\begin{array}{l}\text { DAGOGÍA } \\
\text { ción ECTS) }\end{array}$ & 75 & 3 & Grupo A, B y C & Grupo C & 41 \\
\hline & Edu & ión Infantil & 300 & 5 & Grupo A,B,C,D y E & Grupo A & 60 \\
\hline 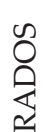 & Educ & ón Primaria & 660 & 11 & $\begin{array}{r}\text { Grupo } \\
\mathrm{A}, \mathrm{B}, \mathrm{C}, \mathrm{D}, \mathrm{E}, \mathrm{F}, \mathrm{G}, \mathrm{H}, \mathrm{I}, \mathrm{J} \\
\text { y K }\end{array}$ & $\begin{array}{l}\text { Grupo } \\
\text { C, E y L }\end{array}$ & 175 \\
\hline & & ación Social & 120 & 2 & Grupo A y B & Grupo B & 44 \\
\hline & & Pedagogía & 120 & 2 & Grupo A y B & Grupo B & 60 \\
\hline & & TOTAL LRU & & & & & 178 \\
\hline & SUBTOTAL EX & $\begin{array}{r}\text { rimentación } \\
\text { ECTS }\end{array}$ & & & & & 131 \\
\hline & SUB & TAL Grados & & & & & 339 \\
\hline & & & & & & TO' & IL: 648 \\
\hline
\end{tabular}

${ }^{a}$ En negrita los grupos seleccionados. ${ }^{\mathrm{b}}$ La muestra productora de datos, representa a un grupo del cluster seleccionado. 
TABLA 2

GUÍAS Y MATERIAS DE LAS TITULACIONES SELECCIONADAS

\begin{tabular}{|l|c|}
\hline \multicolumn{1}{|c|}{ Titulación, Especialidad, Características } & $\mathbf{N}^{\mathbf{0}}$ \\
\hline Educación Primaria LRU & 20 \\
\hline Educación Infantil Experimentación ECTS & 19 \\
\hline Educación Infantil LRU & 16 \\
\hline Pedagogía LRU & 19 \\
\hline Psicopedagogía ECTS & 8 \\
\hline Grado Educación Infantil & 10 \\
\hline Grado Educación Primaria & 9 \\
\hline Grado Educación Social & 10 \\
\hline Grado Pedagogía & 10 \\
\hline TOTAL & 135 \\
\hline
\end{tabular}

a'Ley Orgánica de Reforma Universitaria, 11/1983, de 25 de agosto. B.O.E. de 1 de septiembre. ${ }^{\text {bSe analizan }}$ los programas LRU de la especialidad de Educación Infantil, puesto que pueden aportar datos sobre la evolución o cambio de elementos en los nuevos programas.

\section{FUENTES DE INFORMACIÓN. CARACTERÍSTICASTÉCNICAS DE LOS INS- TRUMENTOS EMPLEADOS}

En la recogida de datos se han empleado diversas fuentes de información directa e indirecta.

\section{I. Fuentes de información directa}

Para identificar el Enfoque de aprendizaje, el instrumento seleccionado se denomina "Cuestionario de Procesos de Estudio" (R-SPQ-2F). Dicho cuestionario está compuesto por un total 20 ítems, basados en las estrategias y motivos que el alumnado declara emplear en su aprendizaje académico. Estos ítems, a su vez, se distribuyen en dos dimensiones de 10 ítems cada una. La primera dimensión denominada Enfoque Superficial y la segunda, Enfoque Profundo.

Debido a la importancia del instrumento en la investigación realizada, se ha considerado necesario realizar un análisis de las propiedades psicométricas del mismo para compararlas con el original (Biggs, Kember y Leung, 2001) y establecer las semejanzas o divergencias de resultados.

Antes de realizar el proceso de factorización, se ha determinado el grado de adecuación de los valores obtenidos. Para ello se emplea los indicadores:

a. En la matriz de correlaciones entre cada variable o ítems, el "determinante" es de 2,939E-05. Lo que indica la existencia de variables con correlaciones entre sí muy elevadas, y por lo tanto, es posible la realización del análisis factorial.

b. Prueba de esfericidad de Barlett. El valor de $\chi^{2}$ es de 787,822 que para g.l. 190, es significativo al 0,000, según esto se puede rechazar la hipótesis nula de que las variables utilizadas en el análisis no se correlacionan en la población de la 
que hemos extraído la muestra. Esto nos permite considerar la matriz de correlaciones $\mathrm{R}$ adecuada para la factorización.

c. El valor de conjunto obtenido en la prueba de $\mathrm{KMO}=0,795$, o lo que es lo mismo, una medida "meritoria" según Kaisser (1974), para seguir con el análisis factorial.

d. Los valores de la diagonal en la matriz de correlación anti-imagen se sitúan entorno al "valor de conjunto", y no existe ninguna variable que quede por debajo del 0,5. Además, fuera de la diagonal principal, son bajos los valores, apenas si se aproximan a 0,25.

e. Y por último, para estimar el grado de ajuste de los datos al modelo se han analizado los residuales o las diferencias entre las correlaciones observadas y las estimadas.

Una vez concluido el análisis de las condiciones de aplicación del análisis factorial, se puede decir que la matriz de correlaciones supera suficientemente las condiciones para que pueda realizarse este análisis, y por lo tanto a continuación se realiza dicho proceso. La determinación de comunalidades, es el primer paso, puesto

TABLA 3A

TABLA 3B

COMUNALIDADES

MATRIZ DE COMPONENTES ROTADOS*

\begin{tabular}{|c|c|c|c|c|c|c|c|}
\hline ÍTEM & Inicial & Extracción & ÍTEM & Factor 1 & Factor 2 & Factor 3 & Factor 4 \\
\hline Ítem 1 & 1,000 & 0,599 & Ítem 1 & 0,528 & & & 0,472 \\
\hline Ítem 2 & 1,000 & 0,616 & Ítem 2 & 0,543 & & & 0,508 \\
\hline Ítem 3 & 1,000 & 0,468 & Ítem 3 & & 0,683 & & \\
\hline Ítem 4 & 1,000 & 0,677 & Ítem 4 & & 0,446 & 0,341 & 0,587 \\
\hline Ítem 5 & 1,000 & 0,753 & Ítem 5 & 0,326 & & & 0,784 \\
\hline Ítem 6 & 1,000 & 0,660 & Ítem 6 & 0,490 & & & \\
\hline Ítem 7 & 1,000 & 0,416 & Ítem 7 & & 0,353 & & \\
\hline Ítem 8 & 1,000 & 0,672 & Ítem 8 & & 0,803 & & \\
\hline Ítem 9 & 1,000 & 0,575 & Ítem 9 & 0,356 & & $-0,428$ & 0,365 \\
\hline Ítem 10 & 1,000 & 0,548 & Ítem 10 & 0,753 & & & \\
\hline Ítem 11 & 1,000 & 0,797 & Ítem 11 & & 0,862 & & \\
\hline Ítem 12 & 1,000 & 0,628 & Ítem 12 & & 0,476 & 0,433 & 0,449 \\
\hline Ítem 13 & 1,000 & 0,613 & Ítem 13 & 0,724 & & & \\
\hline Ítem 14 & 1,000 & 0,680 & Ítem 14 & 0,720 & & & \\
\hline Ítem 15 & 1,000 & 0,690 & Ítem 15 & & 0,698 & 0,387 & \\
\hline Ítem 16 & 1,000 & 0,499 & Ítem 16 & & 0,319 & 0,595 & \\
\hline Ítem 17 & 1,000 & 0,700 & Ítem 17 & 0,748 & & & \\
\hline Ítem 18 & 1,000 & 0,715 & Ítem 18 & 0,837 & & & \\
\hline Ítem 19 & 1,000 & 0,486 & Ítem 19 & & 0,484 & 0,545 & \\
\hline Ítem 20 & 1,000 & 0,584 & Ítem 20 & & & 0,829 & \\
\hline
\end{tabular}

*Método de extracción: Análisis de componentes principales, eliminando pesos $<0.30$. Método de rotación: Normalización Equamax con Kaiser. La rotación ha convergido en 8 iteraciones. 
que determina la proporción de varianza explicada por los componentes. Para este caso, los 20 ítems son explicados por los componentes, puesto que no hay valores próximos a cero, sino que oscilan entre 0,468 y 0,797 (Tabla 3a). Con el método de extracción de los componentes principales, se obtienen cuatro factores que explican el $61,877 \%$ el total de la varianza.

El criterio que se va a emplear para determinar el límite del grado de correlación exigible entre variable y factor, es el que propone Comrey (1985) de 0,3. Una vez realizado el proceso de rotación, y atendiendo al contenido de los ítems con pesos representativos, se comprueba cómo estos corresponden con las cuatro dimensiones (Tabla 3b) que propone Biggs, Kember y Leung (2001) en su cuestionario original, y junto a estas, subyacen dos factores latentes ortogonales: Enfoque Superficial (SA) y Enfoque Profundo (DA).

La fiabilidad global ha sido extraída con el coeficiente alpha de Cronbach, siendo este de 0,709, muy cercano al obtenido por Hernández (2001) en su contexto.

\subsection{Fuentes de información indirecta}

4.2. I. Programas de las materias troncales y obligatorias/básicas de las titulaciones de Grado/Diplomaturas $L R U$ y en experimentación/Licenciaturas $L R U$ y en experimentación

Como fuente de recogida de datos indirecta se han utilizado las guías de las materias troncales y obligatorias/básicas, de las titulaciones seleccionadas, por ser estas las que vertebran el currículo. A través de estos programas hemos identificado las principales características de los modelos metodológicos docentes.

Para ello se realiza un análisis de estos documentos, sometidos a juicio de dos expertos en investigación educativa. Tras la lectura detallada y un análisis del contenido de cada guía de forma pormenorizada, se calcula el grado de acuerdo en la codificación realizada para garantizar la objetividad y la fiabilidad interjueces (Anguera, 1978). Aunque existen diversas formas de concretar la fiabilidad de observaciones (Buendía, Colás y Hernández, 1998; Mitchell, 1978), para nuestro estudio hemos utilizado el porcentaje de concordancia ínter expertos corregido con el índice de Kappa de Cohen, obteniendo un valor de $\mathrm{K}=0,64$ con una $\mathrm{P}_{\mathrm{e}}=0,246$ y $\mathrm{P}_{\mathrm{o}}=0,73$. Estos acuerdos los hemos considerado "acuerdos excelentes" (Landis y Koch, 1977).

\section{ANÁLISIS DE DATOSY RESULTADOS}

Para el análisis de datos y obtención de resultados vamos a seguir la secuencia de los objetivos planteados al principio de nuestro trabajo.

\section{I. Comparación del Enfoque de aprendizaje y características generales de los grupos de diplomaturas: Educación Infantil (Experimentación ECTS) y Educa- ción Primaria (LRU)}

La mayoría del alumnado del grado de Educación infantil tienen 21 años y en el de Educación Primaria 20 años, siendo la calificación media en ambos grupos 2,51 
equivalente a notable medio-alto. Son mujeres un 85,2 \% en el grupo de Educación Infantil, y en el de Educación Primaria un 76,1\%.

El grupo de Educación Infantil, se sitúa preferentemente en el Enfoque Profundo, con una media de 31,65 frente a 23,36 en el Enfoque Superficial. Sin embargo en el grupo de Educación Primaria, el enfoque predominante es el Superficial con una media de 28,69 frente a 26,42 que representa el Enfoque Profundo. En ambos casos, las medias coinciden con los valores en las frecuencias totales. Dichas puntuaciones se reflejan en la Tabla 4 y Figura 1, para cada uno de los Enfoques de aprendizaje: Enfoque Superficial y Enfoque Profundo.

TABLA 4

ENFOQUES DE APRENDIZAJE EN LOS GRUPOS DE GRADO

\begin{tabular}{|l|l|r|r|r|r|r|r|}
\hline \multicolumn{2}{|c|}{ Titulacion y especialidad } & \multicolumn{1}{c|}{ SS } & \multicolumn{1}{c|}{ SM } & \multicolumn{1}{c|}{ DS } & DM & \multicolumn{1}{c|}{ SA } & \multicolumn{1}{c|}{ DA } \\
\hline \multirow{3}{*}{$\begin{array}{l}\text { Educación } \\
\text { Infantil }\end{array}$} & Media & 13,28 & 10,08 & 15,11 & 16,53 & 23,36 & \multicolumn{1}{c|}{31,65} \\
\cline { 2 - 8 } & $\mathrm{N}$ & 88 & 88 & 88 & 88 & 88 & 88 \\
\cline { 2 - 8 } & Desv. Típ. & 3,683 & 3,806 & 3,385 & 3,556 & 6,867 & 6,305 \\
\hline \multirow{3}{*}{$\begin{array}{l}\text { Educación } \\
\text { Primaria }\end{array}$} & Media & 15,96 & 12,73 & 12,31 & 14,11 & & 26,42 \\
\cline { 2 - 8 } & $\mathrm{N}$ & 90 & 90 & 90 & 90 & 90 & 90 \\
\cline { 2 - 8 } & Desv. Típ. & 3,528 & 3,798 & 3,111 & 3,114 & 6,577 & 5,399 \\
\hline \multirow{4}{*}{\begin{tabular}{l} 
Total \\
\cline { 2 - 8 }
\end{tabular}} & Media & 14,63 & 11,42 & 13,70 & 15,31 & 26,06 & $\mathbf{2 9 , 0 1}$ \\
\cline { 2 - 8 } & $\mathrm{N}$ & 178 & 178 & 178 & 178 & 178 & 178 \\
\cline { 2 - 8 } & Desv. Típ. & 3,837 & 4,018 & 3,532 & 3,545 & 7,215 & 6,408 \\
\hline
\end{tabular}

Nota. Se emplean sus iniciales anglosajonas: SA para el Enfoque Superficial; SS para la estrategia superficial y SM para el motivo superficial; DA para el Enfoque Profundo; DS para la estrategia profunda y DM para el motivo profundo.

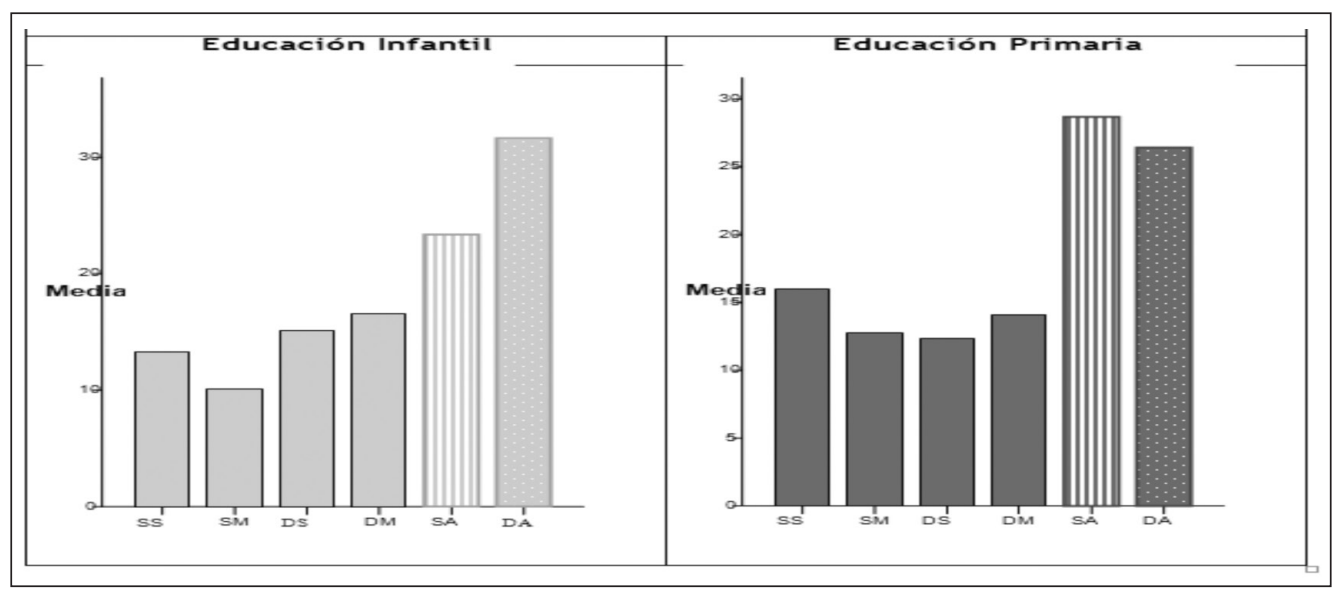

FIGURA 1

ENFOQUES DE APRENDIZAJE: ESTRATEGIAS Y MOTIVOS EN EDUCACIÓN INFANTIL Y EDUCACIÓN PRIMARIA 


\subsection{Comparación entre los Enfoques de aprendizaje y características generales de los grupos de licenciatura: Pedagogía (LRU) y Psicopedagogía (Experimentación)}

Este alumnado de licenciatura tiene una edad comprendida entre los 22 años, en Pedagogía, y 24 años en Psicopedagogía. Aunque en esta última aparece con un índice tres veces mayor de desviación típica $(6,079)$, o lo que es lo mismo, la variabilidad de

TABLA 5

ENFOQUES DE APRENDIZAJE EN LOS GRUPOS DE LICENCIATURA

\begin{tabular}{|c|c|c|c|c|}
\hline Pedagogía & $\mathbf{F}$ & Media & Desv. típ. & Varianza \\
\hline Estrategia Superficial & & 13,56 & 3,119 & 9,726 \\
\hline Motivo Superficial & & 14,07 & 2,743 & 7,524 \\
\hline Estrategia Profunda & & 14,63 & 2,822 & 7,965 \\
\hline Motivo Profundo & & 15,63 & 3,199 & 10,235 \\
\hline Enfoque Superficial & 33 & 27,63 & 4,689 & 21,986 \\
\hline Enfoque Profundo & 56 & 30,27 & 4,989 & 24,894 \\
\hline Psicopedagogía & $\mathbf{F}$ & Media & Desv. típ. & Varianza \\
\hline Estrategia Superficial & & 12,38 & 4,322 & 18,681 \\
\hline Motivo Superficial & & 9,45 & 3,156 & 9,961 \\
\hline Estrategia Profunda & & 15,55 & 4,145 & 17,181 \\
\hline Motivo Profundo & & 16,00 & 3,060 & 9,366 \\
\hline Enfoque Superficial & 5 & 21,83 & 6,724 & 45,215 \\
\hline Enfoque Profundo & 37 & 31,55 & 6,512 & 42,400 \\
\hline
\end{tabular}

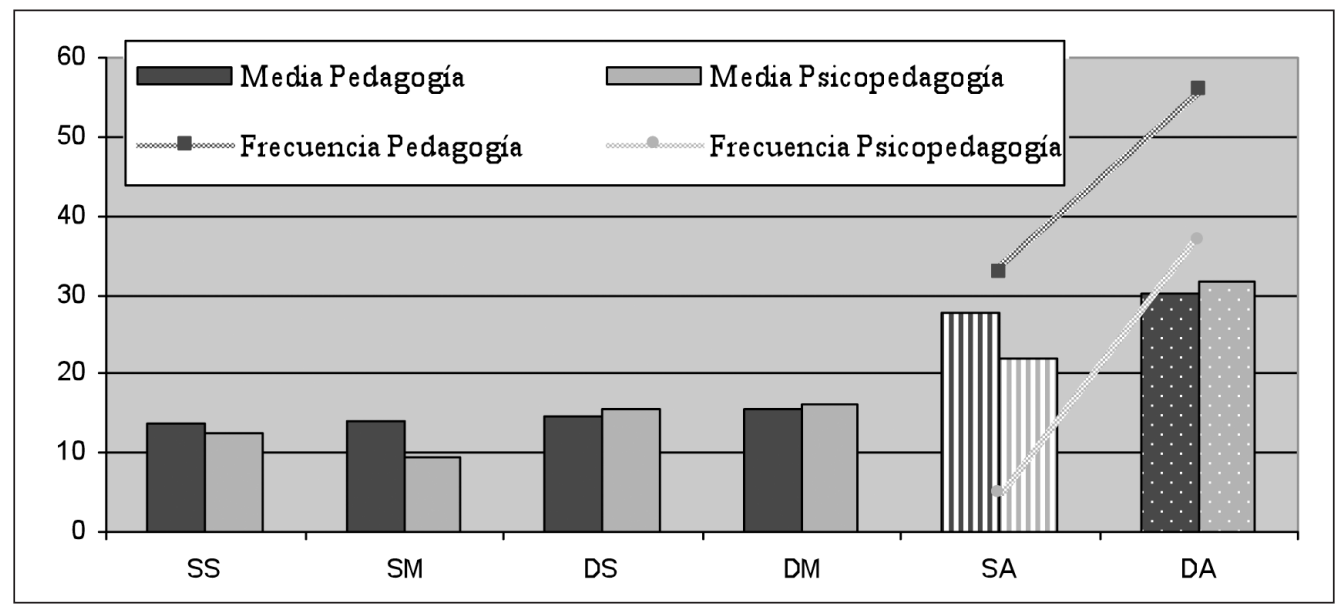

FIGURA 2

ENFOQUES DE APRENDIZAJE: ESTRATEGIAS Y MOTIVOS EN PEDAGOGÍA Y PSICOPEDAGOGÍA 
edad en este grupo se triplica frente a la del grupo de Pedagogía. Por otro lado, su calificación media de 2,49, o lo que en términos cualitativos representa a un "notable medio-alto". Las medias próximas al valor 2 (1=hombre y $2=$ =mujer), nos indica que existe una mayoría mujeres, en ambas titulaciones.

El grupo de Pedagogía, se sitúa preferentemente en el Enfoque Profundo, con una media de 30,27 y frecuencia de 56, para 90 sujetos. Al igual que ocurre en el de Psicopedagogía con media superior en el Enfoque Profundo 31,55 y frecuencia de 37, para 42 sujetos (Tabla 5 y Figura 2).

\subsection{Enfoques de aprendizaje en los nuevos Grados de Educación Infantil, Educa- ción Primaria, Educación Social y Pedagogía}

Los grupos encuestados de los nuevos Grados, tienen edades comprendidas entre los 20 y los 23 años. La calificación media es de 2,22, o lo que es lo mismo "notable medio-alto" en términos cualitativos. En cuanto a la variable género, tenemos una media global de 1,68 lo que indica un mayor número de mujeres que de hombres. Aunque cabe destacar que en el grado de Educación Infantil esta media asciende hasta el 1,93 y sin embargo en el Grado de Pedagogía desciende al 1,5 (Figura 3).
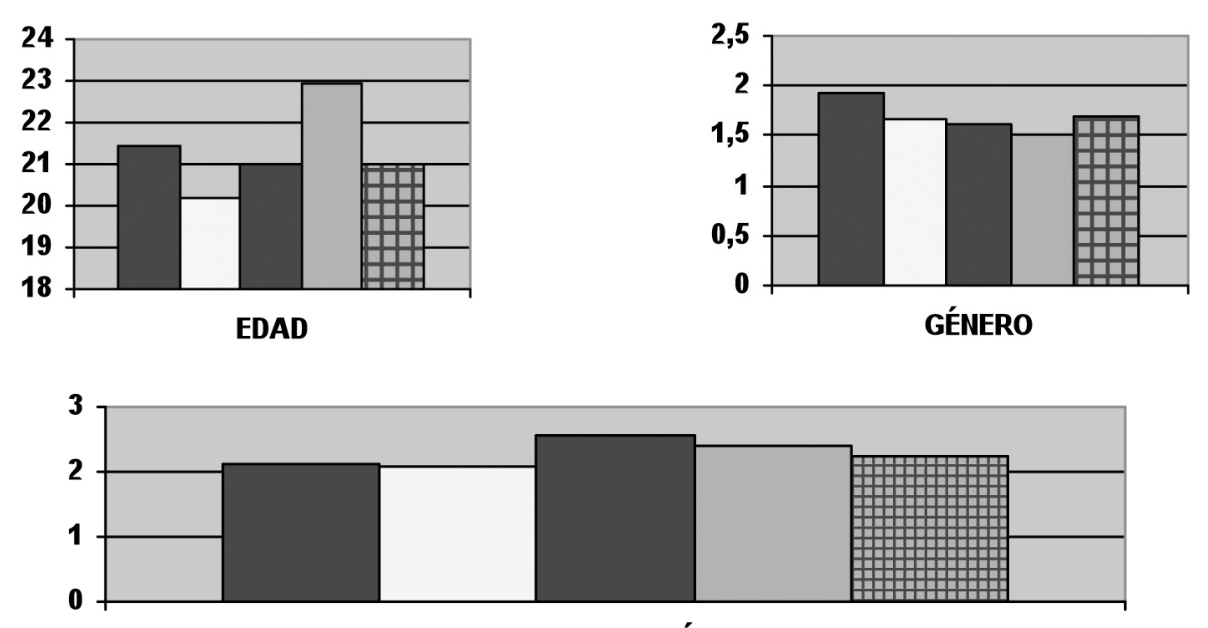

CALIFICACIÓN

Educación Infantil $\square$ Educación Primaria $\square$ Educación Social $\square$ Pedagogía $\square$ Total

FIGURA 3

EDAD, GÉNERO Y CALIFICACIONES MEDIAS EN LOS GRADOS

El Enfoque de aprendizaje, del alumnado de los grupos de Grados, está claramente delimitado en Enfoque Profundo, ya que la distancia entre medias en ambos enfoques es alta y las desviaciones típicas son bajas. Y si comparamos las frecuencias y porcentajes 
obtenidos en ambos enfoques por los cuatro Grados, no se observan variaciones entre estos, lo que nos indica que los grupos están claramente definidos.

Aunque si realizamos un estudio de las subdimensiones, comprobamos que en el grado de Educación Primaria aparece una media un tanto más alta en las Estrategias Superficiales que en las Profundas, siendo la titulación que más débilmente se posiciona en el Enfoque Profundo (Tabla 6 y Figura 4).

TABLA 6

MEDIAS Y FRECUENCIAS EN LAS SUB DIMENSIONES Y DIMENSIONES

\begin{tabular}{|c|c|c|c|c|c|c|c|}
\hline \multicolumn{2}{|c|}{ Titulación y especialidad } & sS & SM & SA & DS & DM & DA \\
\hline \multirow{3}{*}{$\begin{array}{l}\text { Ed. } \\
\text { Infantil }\end{array}$} & Media & 13,2333 & 10,5500 & 23,7833 & 15,7500 & 17,3051 & 33,0678 \\
\hline & Frecuencia & & & 13 & & & 44 \\
\hline & Desv. típ. & 3,23836 & 4,39347 & 7,17598 & 2,78388 & 3,64008 & 5,87180 \\
\hline \multirow{3}{*}{$\begin{array}{l}\text { Ed. } \\
\text { Primaria }\end{array}$} & Media & 14,6207 & 11,0747 & 25,6590 & 14,1886 & 15,5714 & 29,7600 \\
\hline & Frecuencia & & & 61 & & & 109 \\
\hline & Desv. típ. & 3,48487 & 3,86030 & 6,70078 & 3,41462 & 3,39612 & 6,42012 \\
\hline \multirow{3}{*}{ Ed. Social } & Media & 13,1364 & 11,3409 & 24,4773 & 17,2045 & 18,3636 & 35,5682 \\
\hline & Frecuencia & & & 11 & & & 30 \\
\hline & Desv. típ. & 3,41400 & 5,18470 & 8,45917 & 2,00673 & 3,10357 & 4,69734 \\
\hline \multirow{3}{*}{ Pedagogía } & Media & 12,8000 & 11,1000 & 23,9000 & 17,5500 & 18,9500 & 36,5000 \\
\hline & Frecuencia & & & 18 & & & 36 \\
\hline & Desv. típ. & 4,23784 & 5,99915 & 10,08893 & 3,06663 & 2,84292 & 5,26421 \\
\hline \multirow{3}{*}{ Total } & Media & 13,8580 & 11,0207 & 24,8576 & 15,4513 & 16,8373 & 32,2899 \\
\hline & $\mathrm{N}$ & & & 103 & & & 219 \\
\hline & Desv. típ. & 3,65224 & 4,56241 & 7,73158 & 3,39594 & 3,58452 & 6,54761 \\
\hline
\end{tabular}

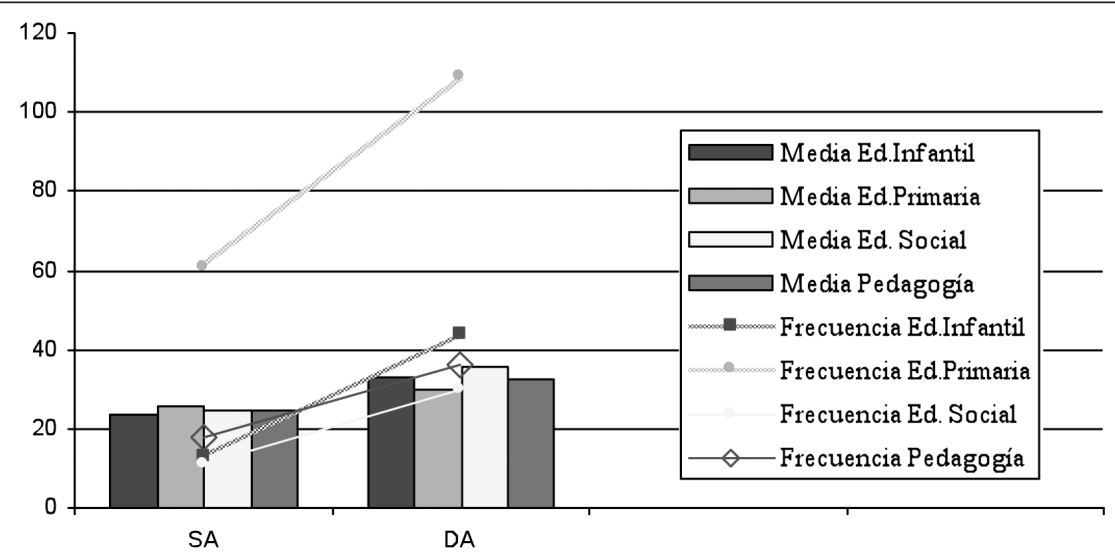

FIGURA 4

FRECUENCIAS Y MEDIAS DE ENFOQUES DE APRENDIZAJE EN LOS GRADOS 


\subsection{Identificación del modelo metodológico reflejado en las guías docentes en función de la titulación}

Considerando los datos obtenidos en el apartado anterior, es necesario realizar un análisis de las guías propias a cada sistema de formación, un total de 123, que suponen el $87,5 \%$ del total.

\subsection{Primaria LRU/Infantil ECTS/Grados Infantil, Primaria y Educación Social}

La primera comparación se realiza entre las diplomaturas con el sistema LRU, las de experimentación ECTS y los nuevos Grados. En los dos primeros grupos no se aprecian variaciones en los supuestos epistemológicos de sus programas; tradicional con tendencia al espontaneísta. El cambio sustancial aparece en los nuevos Grados con un marcado modelo metodológico constructivista. Al igual que ocurre con el concepto de "aprender", el de "enseñanza" y los procesos comunicativos en el aula.

Los roles que adoptan en clase el profesorado y el alumnado están muy relacionados, a priori, en cuanto el modelo empleado, por lo que se hace un análisis conjunto. En los programas LRU la principal figura del profesorado respondía a condicionamientos tecnológicos (70\%) en los que el profesorado se convertía en un planificador de las tareas. Sin embargo, en los modelos de experimentación ECTS y en los nuevos Grados comparte este rol con un modelo espontaneísta, en el que el profesorado deja más "hacer" al alumnado.

En los criterios empleados para seleccionar y organizar los contenidos a desarrollar en los programas, usualmente no son considerados. Sin embargo, en los programas con experimentación ECTS, aunque basados en modelos tradicionales, se comienza a especificar y justificar los motivos de la selección de los contenidos de aprendizaje. Y dicha especificación se hace algo más visible en las guías de los nuevos Grados.

La evaluación sigue siendo uno de los apartados que usualmente más preocupan a los estudiantes. Por lo que en toda la muestra analizada, se explicitan los procedimientos e instrumentos evaluativos, no encontrándose ningún caso que no lo registre.

Igualmente se encuentran pocos casos que fundamenten su evaluación en posicionamientos tradicionales, aunque en los programas LRU hay 5 casos en la parte práctica (25\%), y en los de experimentación ECTS, desaparecen totalmente.

Las distribuciones de frecuencias de los instrumentos de evaluación empleados están muy repartidas en todos los instrumentos registrados. Destacan, en las guías de experimentación ECTS y en los nuevos Grados, el uso variado de distintas técnicas e instrumentos (Figura 5). 


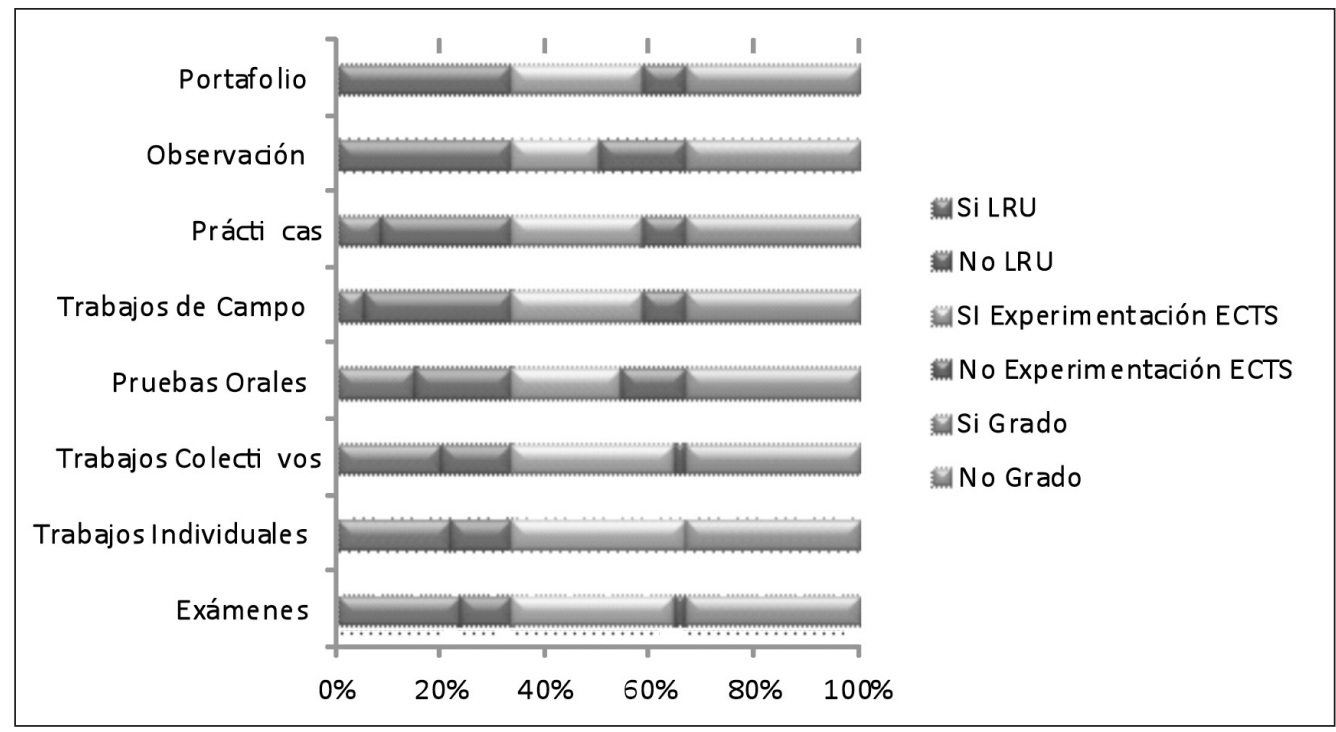

FIGURA 5

INSTRUMENTOS DE EVALUACIÓN EMPLEADOS

\subsubsection{Pedagogía LRU/Psicopedagogía en Experimentación ECTS/Grado de Pedagogía}

La segunda comparación se realiza en las titulaciones de Pedagogía LRU, Psicopedagogía en experimentación ECTS y el nuevo Grado de Pedagogía.

Observamos variaciones en la conceptualización de los "posicionamientos epistemológicos", puesto que en los créditos LRU el 52,6\% se fundamenta en aspectos tradicionales, mientras que en los de experimentación ECTS el mayor porcentaje se sitúa en aspectos tecnológicos. Y en el nuevo Grado de Pedagogía ya se centra en el modelo constructivista casi por completo (90\%).

En lo que se refiere al concepto de "aprender", se puede apreciar que aunque sin grandes distancias en el grupo LRU existe una mayor consideración de posicionamientos tradicionales y en menor grado tecnológicos, en comparación con el grupo en experimentación ECTS. En éste la frecuencia acumulada de los principios tecnológicos y espontaneístas alcanzan el 87,5\% del total. Este desplazamiento culmina en las guías del nuevo Grado de Pedagogía donde ya se centra en el modelo metodológico constructivista plenamente.

Los "criterios empleados para seleccionar y organizar los contenidos" usualmente no se hacen explícitos. El profesorado los considera una cuestión relativa a su labor docente, en la que el alumnado no debe verse implicado. Y si se hacen explícitos algunos criterios para la consideración de unos u otros contenidos, es mayoritariamente desde posicionamientos tradicionales o tecnológicos, en los que la referencia de selección se encuentra en el propio marco disciplinar y ajena a las consideraciones del alumnado o del contexto de aplicación. 
En cuanto al aspecto de cómo se aprecia la formación de las personas, mientras que en los LRU se basan en aspectos tradicionales y tecnológicos ( $\% a>78)$ podemos comprobar un claro desplazamiento en los porcentajes de las guías en experimentación ECTS a posicionamientos más tecnológicos y espontaneístas (\%a=80). Situándose ya casi por completo (80\%) en constructivistas en el nuevo Grado de Pedagogía.

La evaluación de la parte teórica se fundamenta prioritariamente en aspectos de carácter tradicional, en titulaciones con créditos LRU como en titulaciones de experimentación ECTS. No ocurriendo de igual forma en el Grado con posicionamientos evaluativos constructivistas.

Sin embargo, en la evaluación de la parte práctica de las asignaturas si existen variaciones entre las titulaciones LRU vs. Experimentación ECTS. Mientras las primeras siguen basándose en aspectos tradicionales, aunque con porcentajes significativos en tecnológicos y espontaneístas; las segundas se basan claramente en principios de corte constructivista (62,50\%) que junto a los de corte espontaneísta acumulan el $87 \%$ de las frecuencias.

En los instrumentos de evaluación empleados se constata que la evaluación en las asignaturas con créditos LRU se fundamenta habitualmente en la realización de exámenes y trabajos individuales/colectivos. Sin embargo, en los créditos LRU existe una mayor variedad en las pruebas empleadas, siendo destacable comparativamente el mayor empleo de los trabajos de campo y prácticas (Figura 6).

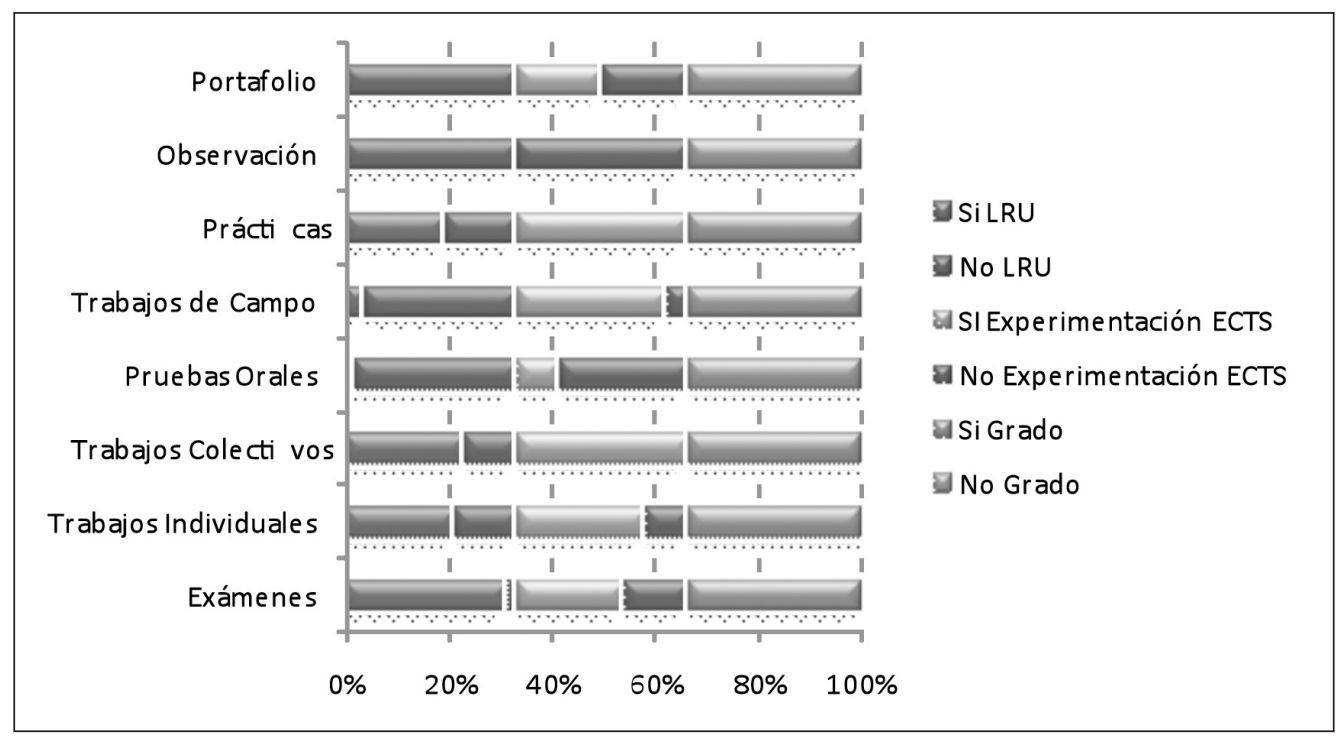

FIGURA 6

INSTRUMENTOS DE EVALUACIÓN EMPLEADOS

Si relacionamos este análisis con los roles esperados de los estudiantes existen ciertas contradicciones. En los créditos LRU se constata el desempeño de roles espontaneístas por parte del alumnado y sin embargo es sometido a pruebas de carácter 
más reproductivo. En los de experimentación ECTS existe una mejor adecuación entre la tipología de pruebas evaluativos empleadas y el rol que se supone deben adoptar los estudiantes, incorporándose instrumentos de evaluación de procesos como son las observaciones de aula y el portafolios. Estos aparecen en las guías del Grado de Pedagogía con el mismo nivel de relevancia que los instrumentos tradicionales; exámenes escritos, orales, trabajos individuales y colectivos.

\section{CONCLUSIONES, HALLAZGOSY LIMITACIONES}

Con los análisis de datos realizados se extraen diversas conclusiones que nos van a ayudar a modificar las estructuras formativas y organizativas para dar respuesta al nuevo modelo propuesto por el EEES.

De manera general, la población de estudiantes analizada queda caracterizada por ser una población con un 75\%-80\% de mujeres en Grados, pero que desciende al 55\% en Psicopedagogía. La edad está en consonancia con los periodos formativos, y las calificaciones son moderadamente altas, situándose en todos los niveles y titulaciones entorno al notable.

Los Enfoques de Aprendizaje en las diplomaturas de Educación Infantil y Primaria, y sus respectivos grados, difieren en función del modelo metodológico docente. En Educación Primaria (LRU) los estudiantes se sitúan en un Enfoque Superficial, frente a los de Infantil (experimentación ECTS), que tienden predominantemente hacia un Enfoque Profundo. Situación que se consolida definitivamente en el Grado de Infantil y con menos intensidad en el Grado de Primaria. El paso intermedio de experimentación ECTS, ha supuesto, según los datos, un puente entre los dos enfoques. Estos posicionamientos del alumnado están en consonancia con el modelo metodológico reflejado a través de las guías docentes, ya que en los Grados encontramos un modelo definitivamente constructivista, mientras que en las titulaciones LRU se sitúan en modelos tradicionales-tecnológicos y las de experimentación ECTS en espontaneístas; el/la profesor/a "dejar hacer" al estudiante, con tendencia hacia los constructivistas en cuanto a la evaluación y los instrumentos empleados para realizarla.

Sin embargo en las licenciaturas, el alumnado se sitúa siempre en un Enfoque Profundo pero con diferencias significativas tanto en las estrategias y como en los motivos. Datos que confirman la situación de estas titulaciones en diversas universidades españolas, situándose los estudiantes de Pedagogía entre los enfoques Profundo y Superficial, y los de Psicopedagogía claramente en un Enfoque Profundo (Hernández Pina y otros, 2001). Esta situación ha sido interpretada por nosotros como resultado del sistema de formación, ya que en Psicopedagogía (con experimentación ECTS) existe una mayor congruencia entre los elementos que caracterizan al modelo en el que se sitúa (espontaneísta con tendencia hacia el constructivista), en comparación con el de Pedagogía (LRU) que pretende un modelo espontaneísta con desarrollos tradicionales. Por lo que se percibe una cierta contradicción entre lo que los profesores/as suelen pensar con lo que suelen hacer, siendo este factor un obstáculo potente para el desarrollo de modelos constructivistas (Porlán, 1996).

Encontramos modelos constructivistas en los nuevos Grados, tanto en los constructos teóricos como son: los "supuestos epistemológicos", el "concepto de aprender", los “cri- 
terios para seleccionar y organizar contenidos", los "conceptos de formación", "enseñar y cómo comunicarse en el aula"; como en aspectos relacionados con la "evaluación". En este sistema tenemos a un alumnado situado claramente en un Enfoque Profundo, lo que nos indica un cierto equilibrio del mismo. Y unas expectativas elevadas de éxito y afianzamiento del nuevo sistema, ya que los estudiantes con Enfoques Profundos se caracterizan por un autoconcepto académico más positivo y una capacidad percibida mayor que le resto. Lo que supone un cierto grado de confianza en sus posibilidades y capacidades que le permiten creer que es capaz de lograr los objetivos (Valle Arias y otros, 2000).

Tras esta comparación entre los modelos metodológicos docentes y los Enfoques de aprendizaje, se confirma que existen, principalmente en los Grado, fuertes conexiones entre las competencias, objetivos y contenidos marcados en el currículo y la metodología seguida por el profesorado, incluida la evaluación realizada. Pero aunque este análisis ha sido exhaustivo y amplio, se ha detectado la necesidad de profundizar tanto en aspectos relacionados con la denominada metáfora de la matrioska (González, Del Rincón, y Del Rincón, 2011), para poder así verificar la consolidación del Enfoque Profundo como aquel que contiene el Enfoque Superficial, siendo este la base del aprendizaje del alumnado. Como en las premisas expuestas en las guías de las materias, donde se detecta una fuerte uniformidad de las guías docentes de los nuevos Grados.

El seguimiento de dicha implementación nos confirmará los elementos fundamentales que definen el modelo, además de comprender mejor la cultura de aprendizaje de los estudiantes de Ciencias de la Educación y configurar eficazmente un sistema de formación acorde con las premisas del EEES; centrado en el aprendizaje a lo largo de toda la vida.

\section{BIBLIOGRAFÍA}

Anguera, Maㅡ T. (1978). Metodología para la observación en las ciencias humanas. Madrid: Cátedra.

Bakeman, R. y Gottman, J. (1989). Observación de la interacción: Introducción al análisis secuencial. Madrid: Morata.

Biggs, J.B. (1987). Study Process Questionnaire (LPQ) Manual. Melbourne: Australian Council for Educational Research.

Biggs, J.B. (1989). Approaches to the Enhancement of Tertiary Teaching, Higher Education Research and Development, 8(19), 7-25.

Biggs, J.B. (1996). Enhancign teaching through constructive alignment, Higher Education, 32, 347-364.

Biggs, J.B., Kember, D. Y Leung, D. (2001). The revised two-factor Study Process Questionaire: R-SPQ-2F. British Journal of Educational Psychology, 58, 258-265.

Birenbaum, M. (1997). Assesment preferences and their relationship to learning strategies and orientations. Higher Education, 33, 71-84.

Buendía, L., Colás, P. y Hernández, F. (1998). Métodos de investigación en Psicopedagogía. Madrid: McGraw-Hill.

Buendía, L. y Olmedo, E. (2000). Estrategias de aprendizaje y procesos de evaluación en educación universitaria, Bordón, 52(2), 151-163. 
Buendía, L., Olmedo, E. y Pegalajar, M. (2001). Estrategias de aprendizaje en la realización de tareas, en X Congreso Nacional de Modelos de Investigación Educativa. A Coruña: AIDIPE.

Buendía, L. y Olmedo, E. (2003). Estudio transcultural de los enfoques de aprendizaje en educación superior. Revista de Investigación Educativa, 21(2), 371-386.

Buendía, L., Olmedo, E., González, G. y Yañez, M. (2005). Approaches to Learning and Peer Task Resolution. International Journal of Learning, 12(4), 349-358.

Buendía, L., Olmedo, E. y González, G. (2009). Lifelong learning: diferentes contextos, diferentes situaciones. Revista de Investigación Educativa, 29(1), 185-203.

Cano, F., Gea, M., Díaz, M., Berbén, A.B.G., y Fernández, M. (2011). Metodología Formativa en el EEES: la Experiencia Internacional de los Estudiantes Erasmus. Memoria del Proyecto de Innovación Docente № 08-240. Universidad de Granada: Granada.

CIDUA (2005). Informe sobre innovación de la docencia en las universidades andaluzas. Sevilla: Junta de Andalucía.

Cole, N.S. (1990). Conceptions of educational achievement, Educational Researcher, 19(3), 2-7.

Comrey, A. (1985). Manual de análisis factorial. Madrid: Cátedra.

CRMEU (2006). Propuesta para la renovación de las metodologías educativas en la universidad. Madrid: MEC.

Dahlin, B. y Watkins, D. (2000), The role of repetition in the processes of memorising and understanding: A comparison of the views of German and Chinese secondary school students in Hong Kong. British Journal of Educational Psychology, 70(1), 65-84.

De Miguel, M. (coord.) (2005). Modalidades de enseñanza centradas en el desarrollo de competencias. Orientaciones para promover el cambio metodológico en el EEES. Oviedo: Ediciones de la Universidad de Oviedo.

De Miguel, M. (coord.) (2006). Modalidades de enseñanza y aprendizaje para el desarrollo de competencias. Madrid: Alianza.

Eklund-Myrskog, G. (1998). Students' conceptions of learning in different educational contexts, Higher Education, 35, 299-316.

González, J.J., Del Rincón, B. y Del Rincón, D. (2011). Estructura latente y consistencia interna del R-SPQ-2F: Reinterpretando los enfoques de aprendizaje en el EEES. Revista de Investigación Educativa, 29(2), 277-294.

Hernández, F. (2000). La evaluación de estudiantes, Jornadas de Medición y Evaluación Educativas: Estándares e Indicadores para Analizar la Realidad Educativa. Universidad de Valencia.

Hernández, F. (2001). Los enfoques de aprendizaje en los estudiantes españoles universitarios. Revista de Investigación Educativa, 19(2), 465-489.

Hernández, F., Martínez, P., Rubio, M. y Rosario, P. (2005). Aprendizaje, competencias y rendimiento en Educación Superior. Madrid: La Muralla.

Jonassen, D. y Rorher-Murphy, L. (1999). Activity Theory as a framework for designing constructivist learning environments. Educational Technology:Research and Development, 46(1).

Landis, J.R. y Koch, G.G. (1977). The measurement of observer agreement for categorical data. Biometric, 33, 159-174. 
Mitchell, S. K. (1978). Interobserver agreement, reliability and generalizability of data collected in observational studies. Psychological Bulletin, 86(2), 376-390.

Porlán, R. (1996). Cambiar la escuela. Buenos Aires: Magisterio del Río de la Plata.

Porlán, R., Rivero, A. y Martín, R. (1998). Conocimiento profesional y epistemología de los profesores, II: Estudios empíricos y conclusiones. Investigación Didáctica, 16(2), 271-288.

Scardamalia, M., Bereiter, C. y Lamon, M. (1994). The CSILE Project : Trying to Bring the Classroom into World 3. En K. McGilly (Ed.), Classroom Lessons: Integrating Cognitive Theory and Classroom Practice. Cambridge: MIT Press, 201.

Valle Arias, R. y otros (2000). Enfoques de aprendizaje en estudiantes universitarios. Psicothema, 12(3), 368-375.

Fecha de recepción: 25 de julio de 2011.

Fecha de revisión: 30 de julio de 2011.

Fecha de aceptación: 31 de mayo de 2012. 
